\title{
Quantum Dots: A Quantum Jump for Molecular Imaging?
}

$\mathbf{V}_{\text {i }}$ a fundamental subject of basic and applied biologic research. In such fields as genetics, biochemistry, and molecular biology, visible fluorescent protein constructs provide researchers with a tool of enormous value that has facilitated the identification of key molecules involved in cellular function in tissue sections and in living cells. Recently, the success of fluorescent methods in biologic research has instigated vast efforts to develop new fluorescent probes for molecular imaging of key cellular molecules in vitro and in vivo.

Conventional fluorophores are organic substances composed of either

\section{See page 1511}

chemically synthesized fluorescent dyes or genetically encoded fluorescent proteins. Although they have proved greatly useful, these traditional fluorophores have several limitations that render them suboptimal for molecular imaging. This includes short fluorescence duration, narrow excitation, and broad emission bandwidths that result in spectral signal overlap and relatively large size that may interfere with protein function. Accordingly, there has been considerable interest in developing fluorophores with improved optical and chemical characteristics for label-

Received May 6, 2007; revision accepted May 16, 2007.

For correspondence or reprints contact: KyungHan Lee, MD, Department of Nuclear Medicine,

Samsung Medical Center, 50 Ilwondong, Kangnamgu, Seoul, Korea.

E-mail: khleenm@yahoo.co.kr

COPYRIGHT @ 2007 by the Society of Nuclear Medicine, Inc.

DOI: 10.2967/jnumed.107.042069 ing specific molecular imaging probes. Research on nanotechnology—a branch of science devoted to development of techniques at the atomic or nanometer scale-led to the discovery of tiny semiconductor nanoparticles with fascinating light-emitting properties. These particles are called quantum dots (QDs), and their exceptionally superior properties surmount the constraints of traditional fluorophores, thereby offering exciting new opportunities for cellular and in vivo imaging.

QDs are inorganic semiconductor crystals comprised of only tens of thousand atoms with extremely attractive optical properties. The core of QDs, typically made up of cadmium selenide (CdSe), absorbs incident photons and this leads to the generation of electron-hole pairs. The pair then rapidly recombines and there is emission of less-energetic photons. The optical properties of QDs are attributed to quantum confinement - a phenomenon attributed to their nanoscale size, which leads to physical confinement of excitons. This presents QDs with many unique features that favor their biologic use over conventional fluorophores (1). First, fluorescence emitted by QDs is enormously brighter than that of traditional fluorophores. QDs have large extinction coefficients, a measure of the absorbance of incident light per unit concentration of dye and length of path traversed, as well as high quantum yields, a measure of photon quanta emitted over that absorbed. Second, QDs have large absorption coefficients across a wide spectral range. This means that multiple probes with different emissions can be simultaneously excited using a single excitation wavelength. Their emission spectra, in contrast, have very distinct and narrow wavelengths. The size and composition of the nanocrystals can be adjusted to tune the spectrum of light emitted so that QDs of many different colors can be created. Thus, they are well suited for combinatorial optical encoding, in which multiple colored fluorophores are combined to encode thousands of molecules simultaneously. Another advantage of QDs is that they do not easily fade - that is, they are highly resistant to photobleaching, a process in which fluorescence is lost through irreversible alteration of its molecular structure by photodamage, enzymatic degradation, and chemical damage $(2,3)$.

The surface of QDs needs alteration to increase stability and allow conjugation of biomolecules for specific targeting. QD cores are encapsulated by an inert and transparent shell typically consisting of $\mathrm{ZnS}$, which passivates the particle, reduces photochemical bleaching, and increases quantum yield (Fig. 1A). Because preparation in organic solvents results in a hydrophobic organic monolayer, a key advancement was the development of methods to phase-transfer dots grown in organic solvents to water while maintaining a bioactive surface (3). To achieve specific targeting abilities, polymer-coated QDs are covalently linked to biomolecules, including peptides, antibodies, nucleic acids, and small-molecule ligands (Fig. 1B). The availability of such bioconjugation approaches has triggered an explosion in the application of QDs for imaging of molecular targets in living cells and animal models. The first studies to use QDs for labeling proteins in cells were reported less than $10 \mathrm{y}$ ago and used dots conjugated to transferrin (4) and actinbinding molecules ( $(1)$. Other applications that followed include labeling of live cells for multicolor imaging by dots conjugated with an antibody against 


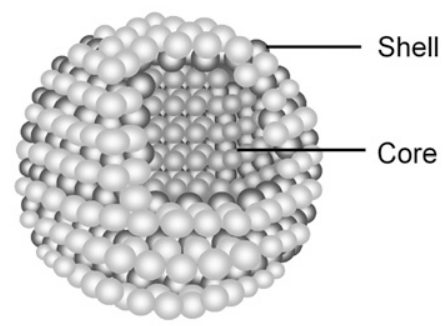

B

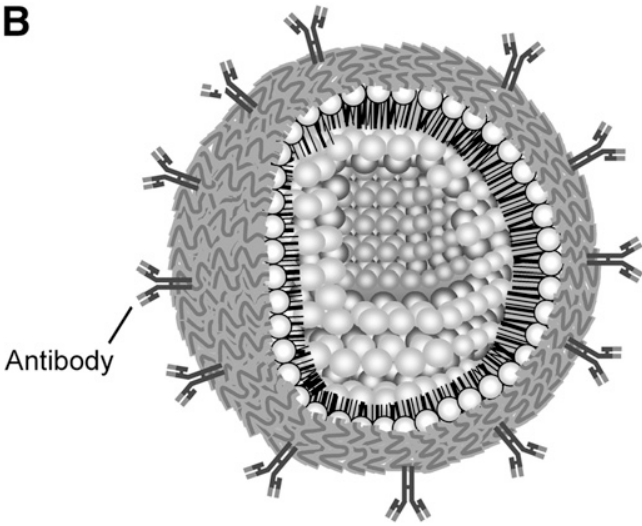

FIGURE 1. (A) Schematic of a semiconductor quantum dot composed of its core and shell structure. (B) Schematic of a quantum dot with polymer-coated surface and conjugation with antibodies for specific targeting.

P-glycoprotein (2), imaging of erbB receptors expressed on live cells with epidermal growth factor-conjugated QDs (5), and HER2 receptor imaging using IgG-conjugated QDs (3). These studies open new avenues for the use of QD probes for investigating such cellular events as ligand-receptor interactions and biomolecular transport.

The photophysical properties of QDs also make them the preferred fluorophores for use in living subjects. Their increased light emission permits brightness in tissues despite severe light attenuation by scattering and absorption. In addition, large separation between excitation and emission peaks allows sensitive detection amid high-background autofluorescence. The first investigation on the feasibility of QDs for use in vivo was reported by Akerman et al. in 2002 (6). In this study, 3 different probes prepared by coupling vasculature-targeting peptides to the surface of QDs injected into xenografted mice showed excellent specificity for targeting lung and tumor vessels (6). Further advances have facilitated the use of QDs for actual molecular imaging in living animals. Larson et al. demonstrated QD imaging of blood flow in capillaries through the skin of living mice (7). Gao et al. introduced a new class of multifunctional QD probes that contain a triblock copolymer for linking tumor-targeting ligands and demonstrated sensitive imaging of cancer cells under in vivo conditions (8). Kim et al. synthesized a novel coreshell nanostructure that emits in the near-infrared spectrum, which allowed clear imaging and subsequent surgical removal of involved sentinel lymph nodes in a pig (9). These studies indicate a potential clinical value of optical imaging with QD probes as an adjunct to radioactive techniques for delineation of pathologic sites during intraoperative procedures. An additional strength of QDs is that they can be used as multiple probes tagged in multicolor fashion to allow tracking of several molecular targets simultaneously, a feature of significant challenge for other imaging techniques.

The versatile polymer coatings of QDs serve as a building block on which multiple functional agents can be assembled. Therefore, it is possible to create multimodality imaging probes by surface integration of paramagnetic or radioactive agents. Indeed, several QD particles have been successfully synthesized for dual-mode optical imaging and MRI. On pages 1511-1518 of this issue of The Journal of Nuclear Medicine, Schipper et al. report an investigation on the in vivo application of radiolabeled QDs (10). The authors prepared 525- and 800-nm emission wavelength $\mathrm{CdSe} / \mathrm{ZnS}$ coreshell QDs, with or without conjugation of 2,000 molecular-weight polyethylene glycol (PEG), and radiolabeled the particles with ${ }^{64} \mathrm{Cu}$. When injected intravenously into nude mice, both larger and smaller QDs showed rapid and high hepatic and splenic uptake without evidence of clearance, as assessed by biodistribution and microPET analysis. PEGylation had the effect of slowing down somewhat the rates of blood clearance and hepatic and splenic uptake of the nanoparticles. The authors conclude that rapid reticuloendothelial system (RES) clearance will require modification of QDs for in vivo utility and that formal quantitative biodistribution and imaging studies will be helpful in their evaluation. QDs have a well-known tendency to accumulate in the RES of the liver and spleen. PEG conjugation is often used to minimize molecular interaction and nonspecific binding, and PEGylation has been indicated to reduce RES elimination and extend the circulation half-life of QDs (11). However, previous studies on the in vivo kinetics of QDs have relied on fluorescence imaging, a method that is severely limited by depth of light penetration and lack of quantitative and tomographic information. On the other hand, the work by Schipper et al. exemplifies the unique ability of radiotracer techniques to provide quantitative in vivo biodistribution and imaging data. Indeed, this article appears to be the first report of quantitative biodistribution of QDs in living mice. More importantly, the study illustrates the huge benefits of combining the positive features of radiotracer methods and nanoparticle technology for molecular imaging. The availability of dual-mode radioactive and optical QD probes could allow correlation of information obtained by PET or SPECT in living subjects with that offered by optical imaging. For instance, the deepimaging capabilities of PET or SPECT could identify a patient's disease site preoperatively, which could then be complemented by fluorescent signals that provide a visual guide to the involved tissues during surgery.

One obstacle for the immediate application of the technology in human subjects is the potential toxicity of QDs. Apprehensions have been raised by indications that $\mathrm{CdSe}$ dots are highly 
toxic to cultured cells under ultraviolet illumination for extended periods of time. This property was attributed to the liberation of toxic cadmium and selenium ions from the core during oxidation. Although several other reports claim that QDs have little, if any, cytotoxic effects $(2,7,9,11)$, further studies must clarify such safety issues before QDs can enter the clinical arena.

QDs are already a valuable tool in basic and applied biology, and recent advances in their in vivo targeting capability are beginning to realize their implementation for diagnostic imaging. The study of Schipper et al. (10) illustrates how unique quantitative information can be added to this promising new nanoparticle technology by integration of radiotracer methods, an integration that will hopefully contribute to a most luminous future for QDs in the field of molecular imaging.

\section{Kyung-Han Lee \\ Samsung Medical Center \\ Seoul, Korea}

\section{REFERENCES}

1. Bruchez M, Moronne M, Gin P, Weiss S, Alivisatos AP. Semiconductor nanocrystals as fluorescent biological labels. Science. 1998;281:2013-2016.

2. Jaiswal JK, Mattoussi H, Mauro JM, Simon SM. Long-term multiple color imaging of live cells using quantum dot bioconjugates. Nat Biotechnol. 2003;21:47-51.

3. Wu X, Liu $\mathrm{H}$, Liu J, et al. Immunofluorescent labeling of cancer marker Her2 and other cellular targets with semiconductor quantum dots. Nat Biotechnol. 2003;21:41-46.
4. Chan WC, Nie S. Quantum dot bioconjugates for ultrasensitive nonisotopic detection. Science. 1998;281:2016-2018.

5. Lidke DS, Nagy P, Heintzmann R, et al. Quantum dot ligands provide new insights into erbB/HER receptor-mediated signal transduction. Nat Biotechnol. 2004;22:198-203.

6. Akerman ME, Chan WC, Laakkonen P, Bhatia SN, Ruoslahti E. Nanocrystal targeting in vivo. Proc Natl Acad Sci USA. 2002;99:12617-12621.

7. Larson DR, Zipfel WR, Williams RM, et al. Watersoluble quantum dots for multiphoton fluorescence imaging in vivo. Science. 2003;300:1434-1436.

8. Gao X, Cui Y, Levenson RM, Chung LW, Nie S. In vivo cancer targeting and imaging with semiconductor quantum dots. Nat Biotechnol. 2004;22:969-976.

9. Kim S, Lim YT, Soltesz EG, et al. Near-infrared fluorescent type II quantum dots for sentinel lymph node mapping. Nat Biotechnol. 2004;22:93-97.

10. Schipper ML, Cheng Z, Lee S-W, et al. microPETbased biodistribution of quantum dots in living mice. J Nucl Med. 2007;48:1511-1518.

11. Ballou B, Lagerholm BC, Ernst LA, Bruchez MP, Waggoner AS. Noninvasive imaging of quantum dots in mice. Bioconjug Chem. 2004;15:79-86. 